\title{
Zu den ägyptischen Wortspielen mit dem Gottesnamen „Amun“
}

\author{
Stefan Bojowald \\ Bonn, Germany
}

\begin{abstract}
This contribution is dedicated to Egyptian plays on words with the god name "Imn" "Amon". In previous research the question has been dealt with only marginally. Sixteen examples are introduced in the present article. The plays on words can often be traced back to phonetic origins, which are followed in every case.
\end{abstract}

Keywords: Egyptian philology, Egyptian plays on word, Egyptian plays on word with the god name "Imn" "Amon".

Die ägyptische Sprache hält bekanntlich ein reichhaltiges Reservoir an Wortspielen bereit. Die Forschung ist schon früh auf diesen Tatbestand aufmerksam geworden ${ }^{1}$. In der Vergangenheit konnte das

\footnotetext{
${ }^{1} \mathrm{Zu}$ Wortspielen im Ägyptischen vgl. Maria Theresia Derchain-Urtel, Wortspiele zu „Ort“ und „Bewegung“ in Edfu und Dendera, Mélanges Adolph Gutbub, Montpellier 1984, 55-61; Waltraud Guglielmi, $\mathrm{Zu}$ einigen literarischen Funktionen des Wortspiels, Studien zu Sprache und Religion Ägyptens, Band 1, Festschrift für W. Westendorf, Göttingen 1984, 491-506; Waltraud Guglielmi, Lexikon der Ägyptologie VI, 1287-1291, s. v. Wortspiel; Yekaterina Barbash, The Mortuary Papyrus of Padikakem Walters Art Museum 551, Yale Egyptological Studies 8, New Haven 2011, 16ff; Coleen Manassa, The Late Egyptian Underworld: Sarcophagi and Related
} 
diesbezügliche Wissen stetig vertieft und eine Fülle von neuen Erkenntnissen hinzugewonnen werden. Die allgemeine Tendenz scheint bei anderen orientalischen Sprachen in durchaus ähnliche Richtung zu gehen.

In den letzten Jahren ist durch Verf. mit ersten Vorarbeiten zur Untersuchung von ausgewählten Wortspielen begonnen worden. Der vorliegende Beitrag stellt ein weiteres Glied in dieser Kette dar. Im Rahmen dieses Artikels sollen die Wortspiele mit dem ägyptischen Gottesnamen „Imn“ „Amun“ behandelt werden. In die Erörterungen soll zunächst mit einigen allgemeinen Bemerkungen eingeführt werden.

Der Gott Amun hat wohl mit zu den prominentesten Vertretern im ägyptischen Pantheon gehört. Der Aufstieg vom unbedeutenden Lokalgott zum Reichsgott hat sich schon im Mittleren Reich vollzogen. In der Frage der Bedeutung des Namens hat Fecht ${ }^{2}$ wichtige Impulse gesetzt, der die Alternativen „Verborgenheit“, „Verborgener“, „Bleibender“ und „Komme zu mir“ für theoretisch denkbar hält. Die gleichen Begriffe werden unten nicht ganz zufällig als Bestandteile von Wortspielen wiederkehren. Die ,jeux graphiques" bei den Schreibungen des Namens in den ptolemäischen Texten des zweiten Pylons von Karnak sind von Broze/Preys ${ }^{3}$ als Gegenstand gewählt worden. Die religiösen Aspekte sind ebenfalls aus verschiedener Warte betrachtet worden. Die Studie von Sethe ${ }^{4}$ stellt sich darunter immer noch als einschlägig heraus. Die Amuntheologie im frühen Neuen Reich und während der

Texts from the Nectanebid Period, Part 1: Sarcophagi and Texts, Ägypten und Altes Testament 72, 1, Wiesbaden 2007, 9 n. 55; Richard Jasnow/Karl Theodor Zauzich, The ancient Egyptian Book of Thot, A Demotic Discourse on Knowledge and Pendant to the Classical Hermetica, Volume 1: Text, Wiesbaden 2005, 114-117.

${ }^{2}$ Gerhard Fecht, Zum Text der Votivstatue für Amun, publiziert in MDAIK 38, 1982, 334-337, in: Mitteilungen des Deutschen Archäologischen Instituts Abteilung Kairo 40 (1984), 7-11, hier 9.

${ }^{3}$ Michèle Broze/René Preys, Les ,noms cachés“ d' Amon, jeux de signes et rituals sur la porte ptolémaïque du deuxième pylône du temple de Karnak, in: Christiane Zivie-Coche/Ivan Guermeur, „Parcourir 1' Éternité“, Hommages à Jean Yoyotte I, Bibliothèque de 1' École des Hautes Études Sciences Religieuses 156, Turnhout 2012, 183-193.

${ }^{4}$ Kurt Sethe, Amun und die acht Urgötter von Hermopolis, Eine Untersuchung über Ursprung und Wesen des ägyptischen Götterkönigs, Aus den Abhandlungen der Preussischen Akademie der Wissenschaften Jahrgang 1929, Phil.-Hist. Klasse Nr. 4, Berlin 1929, 7-126. 
Ramessidenzeit ist von Assmann ${ }^{5}$ genauer unter die Lupe genommen worden. Die Epitheta des Gottes sind durch Leitz ${ }^{6}$ einer zusammenfassenden Betrachtung gewürdigt worden. Der Niederschlag der Amunvorstellungen auf die religiöse Literatur und Dichtung der Ramessidenzeit hat im Mittelpunkt der Betrachtungen von Oswalt ${ }^{7}$ gestanden. Die Arbeit von Guermeur ${ }^{8}$ sollte bei dieser Gelegenheit ebenfalls nicht unerwähnt bleiben. Die Wortspiele mit „Imn“ „Amun“ sind dagegen nur ganz am Rande zur Sprache gekommen, so dass sich eine größer angelegte Untersuchung durchaus lohnen könnte.

Das Thema wird hier daher zum ersten Mal in etwas ausführlicherer Form behandelt. Die folgende Liste wird insgesamt sechzehn Beispiele umfassen. Die Vorschläge werden mit der Hoffnung unterbreitet, dass wenigstens ein Teil davon die Akzeptanz des Lesers finden wird. Die Thesen sind lediglich als Beitrag zur Diskussion zu verstehen. Die Wortspiele können häufig auf bestimmte Lautgesetze zurückgeführt werden. In vielen Fällen werden sich außerdem Metathesen zeigen. Die Belege werden der Einfachheit halber in alphabetischer Reihenfolge aufgeführt. Die Angaben in den Klammern sollen der groben zeitlichen Einordnung der Belege dienen. Das Alte Reich hat kein einziges Beispiel beigesteuert, was in Anbetracht der obigen Bemerkung zum Auftreten des Gottes kein Wunder ist. Die Blütezeit der Wortspiele hat im Neuen Reich und in der Spätzeit gelegen.

Das erste Wortspiel ist zwischen „Imn“ „Amun“ und „im“ „Meer" erzeugt worden, das durch den Personennamen ,Imn-p3-im“99 (Spätzeit) „Amun vom Meer“ überliefert wird. Das Wort , im“" „Meer“"

\footnotetext{
${ }^{5}$ Jan Assmann, Re und Amun, Die Krise des polytheistischen Weltbildes im Ägypten der 18.-20. Dynastie, Orbis Biblicus et Orientalis 51, Freiburg (Schweiz)/Göttingen 1983, 145ff/189-277.

${ }^{6}$ Christian Leitz (Hrsg.), Lexikon der ägyptischen Götter und Götterbezeichnungen, Band I, $3-y$, Orientalia Lovaniensia Analecta 110, Leuven - Paris - Dudley, MA 2002, 308-320.

${ }^{7}$ John Newell Oswalt, The concept of Amon - Re as reflected in the hymns and prayers of the Ramesside Period, Ann Arbor 1968, 1-294.

${ }^{8}$ Ivan Guermeur, Les cultes $d^{\prime}$ Amon hors de Thèbes. Recherches de géographie religieux, Bibliothèque de 1'École des Hautes Études - Sciences Religieux 123, Turnhout 2005 (non vidi!).

${ }^{9}$ Günther Vittmann, Drei thebanische Urkunden aus dem Jahre 175 v. Chr. (Papyri Louvre E 3440 A + B und Berlin P 3112), Enchoria 15 (1987), 97-146, hier 133.
} 
ist dort als Bezeichnung für das Fayyum verwendet worden. Das Wortspiel kann mit dem Wegfall von , $n^{\prime \prime}$ am Ende des Wortes erklärt werden, zu dem alles Nötige bei Sethe ${ }^{10}$, Westendorf ${ }^{11}$ und JansenWinkeln ${ }^{12}$ gesagt worden ist. Die Einbeziehung von Wortspielen in Personennamen lässt sich übrigens gar nicht so selten beobachten. Die Reihe wird weiter unten durch zwei ähnlich gelagerte Fälle komplettiert.

Das zweite Wortspiel ist zwischen „Imn“ „Amun“ und ,imn“ „verbergen“ abgelaufen, das sich seitens des Ägypters offenbar besonders großer Popularität erfreut hat. Die Erscheinung kann hier an fünf Beispielen verdeutlicht werden. Das erste Beispiel kann in ,ntk Imn, imn=f św $r$ stš $m$ idh.w $n . w \quad s m 3-<b h d . t>$ "13 (Spätzeit) demonstriert werden, wofür die Übersetzung „Du bist Amun, der sich vor Seth in den Sumpfgebieten von Sema-<behedet> verbirgt" am praktischsten sein dürfte. Das zweite Beispiel wird durch die Verbindung ,śdg3 św, iwtỉ ini $\underline{d} r . w=f$, nn rh śs $m-m$ ' mśi.w=f, imn $r n=f s^{\prime} h 3 p$ b3. $w=f, m r n=f$ pwii $n$ 'Imn ${ }^{\text {“14 }}$ (Neues Reich) verkörpert, die sich mit „Der sich versteckt. Dessen Grenzen nicht erreicht werden. Es gibt keinen, der ihn kennt unter seinen Kindern. Der seinen Namen verbirgt, der seine Macht verhüllt, in diesem seinem Namen „Amun“““ wiedergeben lässt. Das dritte Beispiel kann in ,Imn imn $r n=f r$ $n t r . w^{\text {“15 }}$ lokalisiert werden, wofür die Übersetzung „Amun, der seinen Namen verbirgt vor den Göttern" in Anspruch genommen werden

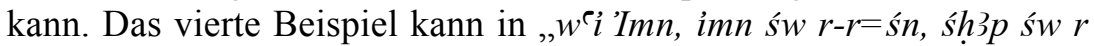
$n \underline{t} r . w^{{ }^{616}}$ identifiziert werden, wofür die Übersetzung „Einer ist Amun, der sich verbirgt vor ihnen, der sich verhüllt vor den Göttern“ am meisten Sinn ergibt. Das fünfte Beispiel kann in ,imn=k tw m 'Imn wr,

\footnotetext{
${ }^{10}$ Sethe, Aegyptisches Verbum, $130 \$ 233$.

${ }^{11}$ Westendorf, Grammatik der medizinischen Texte, 27-28.

${ }^{12}$ Karl Jansen-Winkeln, Spätmittelägyptische Grammatik der Texte der 3. Zwischenzeit, Ägypten und Altes Testament 34, Wiesbaden 1996, 36.

${ }^{13}$ Dieter Kurth, Edfou VIII, Die Inschriften des Tempels von Edfu, Abteilung I, Übersetzungen, Band 1, Wiesbaden 1998, 17.

${ }^{14}$ Jan Zandee, Der Amunshymnus des Papyrus Leiden I 344, verso, Band I, Leiden 1992, 120.

${ }^{15}$ Jan Zandee, De Hymnen aan Amon van Papyrus Leiden I 350, Oudheidkundige Mededelingen uit het Rijksmuseum van Oudheden, Nieuwe Reeks XXVIII, Leiden 1947, 66.

${ }^{16}$ Zandee, Hymnen aan Amon, 75.
} 
$i t n w=k m$ hpr.w=k $m$ itn, $t 3-t n n$ śtnw św $r$ ntr.w $w^{\text {“17 }}$ empor geholt werden, für das die Übersetzung „Du verbirgst dich als (?) der große Amon, du bist ... in deiner Gestalt als Sonnenscheibe, Tatenen, der sich über die Götter erhebt" nahe liegt. Die Grundlage des Wortspiels hat die Homonymie der beiden Wurzeln gebildet. Die Bedeutung „verbergen“ war von Fecht als möglicher Hintergrund des Gottesnamens angesehen worden.

Das dritte Wortspiel hat zwischen „Imn“ „Amun“ und „m3i“" „Löwe“ bestanden, das im Personennamen „m3i 'Imn“"18 (Neues Reich) „Amun ist ein Löwe“ klar vor Augen tritt. Der Lautwandel zwischen

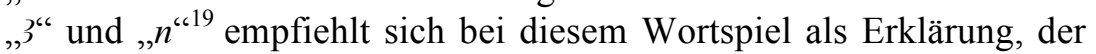
hier mit einer Metathese kombiniert worden ist.

Das vierte Wortspiel hat sich zwischen „Imn“ „Amun“ und

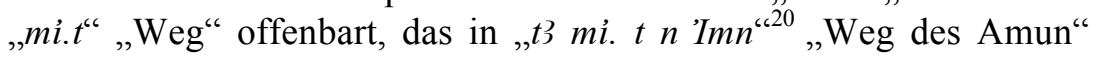
nachvollzogen werden kann. Das Wortspiel ist durch den Abfall von „ $n^{\prime}$ unterstützt worden, der bereits am ersten Beispiel beteiligt war. Das Wortspiel ist zweitens durch eine Metathese zustande gebracht worden. Der betreffende Weg hat laut Grieshaber auf der thebanischen Westseite den Nil mit Medinet Habu verbunden. Im Anschluss werden noch weitere geographische Bezeichnungen folgen, die Wortspiele mit ,Imn“" „Amun“ enthalten.

Das fünfte Wortspiel ist zwischen ,Imn“ „Amun“ und „m in.t" „im Tal“ zustande gekommen, das durch den Personennamen „'Imn$m$-in.t ${ }^{\text {“21 }}$ (Neues Reich/Spätzeit) „Amun ist im Tal“" repräsentiert wird. Die Grundlage des Wortspiels hat sich aus der jeweils unterschiedlichen Verteilung derselben drei Radikale ergeben.

\footnotetext{
${ }^{17}$ H. O. Lange, Der Magische Papyrus Harris, Det Kgl. Danske Videnskabernes Selskab, Historisk-filologiske Meddelelser XIV, 2, Kobenhavn 1927, 33-34.

${ }^{18}$ Hermann Ranke, Die ägyptischen Personennamen, Band I, Verzeichnis der Namen, Glückstadt 1935, 144; Pierre Grandet, Catalogue des ostraca hiératiques non littéraires de Deir El-Médînéh, Tome X $N^{\text {os }}$ 10001-10123, Documents de Fouilles de L'IFAO 46, Le Caire 2006, 7.

${ }^{19}$ Westendorf, Grammatik der medizinischen Texte, 11-12.

${ }^{20}$ Fr. Grieshaber, Lexikographie einer Landschaft, Beiträge zur historischen Topographie Oberägyptens zwischen Theben und Gabel as-Silsila anhand demotischer und griechischer Quellen, GOF IV: Reihe Ägypten 45, Wiesbaden 2004, 44.

${ }^{21}$ Ranke, Personennamen, 2722.
} 
Das sechste Wortspiel hat zwischen „Imn“ „Amun“ und „mi

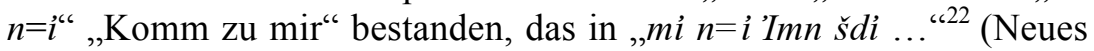
Reich) „Komme zu mir, Amun, und rette...“ tradiert ist. Der Schlüssel zum Verständnis des Wortspiels würde auch hier in der unterschiedlichen Verteilung derselben drei Radikale liegen. Die Bedeutung „Komme zu mir" war von Fecht als möglicher Kern des Gottesnamens vermutet worden.

Das siebte Wortspiel tritt in deutlichen Konturen zwischen „Imn“",Amun“ und „mn“ „,bleiben, dauern“ hervor, das hier an zwei Beispielen illustriert werden kann. Das erste Beispiel kann aus , $i 3 w . t$ rnpi śbb nḥh, 'Imn $m n \quad m$ (i)h.h.wt $n b(. w t)^{\text {“ } 23}$ (Neues Reich) herauspräpariert werden, für das die Übersetzung „du Alter, der du dich verjüngst und die Ewigkeit durchlebst, Amon, der in allem fest ist.“ am weitesten führt. Das zweite Beispiel lässt sich in „,nhb(.t) $[=f]$

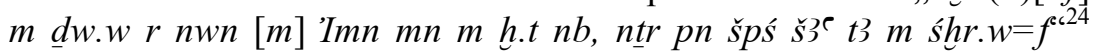
(Spätzeit) feststellen, wofür die Übersetzung ,,[seine] Namensreihe reicht von den Bergen zu der See [als] „Amun, Der Dauert In Allem“, dieser ehrwürdige Gott, der die Welt durch seine Pläne begonnen hat" die beste Wahl darstellt. Die Basis für das Wortspiel ist im Ausfall von $i$ " zu Beginn des Wortes zu finden, zu dem Westendorf einige wichtige Hinweise gegeben hat ${ }^{25}$. Das Wortspiel kann durch die Defektivschreibung „,mn" ${ }^{\text {“26 }}$ für ,Imn“ „Amun“ von einer weiteren Seite abgesichert werden. Die gleiche Erscheinung ist bei den

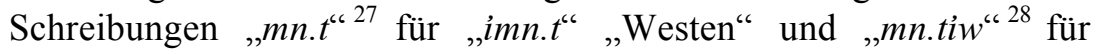
„,imn.tiw“" „westliche (Götter)“" in Kraft getreten. Die Bedeutung „bleiben“ war ebenfalls unter den Vorschlägen von Fecht für den

${ }^{22}$ Jaroslav Černý/Alan H. Gardiner, Hieratic ostraca, Volume I, Oxford 1957, Pl. VIII.

${ }^{23}$ Lange, Magischer Papyrus Harris, 34.

${ }^{24}$ David Klotz, Adoration of the ram, Five hymns to Amun-Re from Hibis Tempel, Yale Egyptological Studies 6, New Haven 2006, 74.

${ }^{25}$ Westendorf, Grammatik der medizinischen Texte, 12/13.

${ }^{26}$ Wolfgang Helck, Urkunden der 18. Dynastie, Übersetzung zu den Heften 17-22, Berlin 1961, 187 n. 4; Karl Jansen-Winkeln, Inschriften der Spätzeit Teil 1: Die 21. Dynastie, Wiesbaden 2007, 188.

${ }^{27}$ Daniel A. Werning, Das Höhlenbuch, Textkritische Edition und Textgrammatik, Teil II: Textkritische Edition und Übersetzung, Göttinger Orientforschungen, IV. Reihe: Ägypten 48, Wiesbaden 2011, 18.

${ }^{28}$ Barbash, Mortuary Papyrus, 152. 
Gottesnamen zu finden. Die Wortspiele würden sich perfekt in dieses Szenario einfügen.

Das achte Wortspiel deutet sich zwischen „Imn“ „Amun“ und „mni.t“ „Landepflock“ an, für das ein sicherer Beleg vorhanden ist. Der locus classicus findet sich in der Passage,,$h^{c}=i$ sf $h r w h r$ c.wi

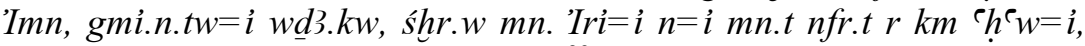
$r d i$ (wi) $n=f r \underline{d} r . w=i$, $n t f$ mni.t $i^{\prime c 29}$ (Neues Reich), für welche die Übersetzung „Ich habe Gestern und Heute in Amuns Hände gelegt, ich war heil befunden und meine Pläne beständig. Ich mache mir ein schönes Bleiben, bis meine Zeit erfüllt ist, indem ich (mich) ihm ganz zu eigen gebe: er ist mein Landepflock" gut zu passen scheint. Der Hauptbeitrag zu diesem Wortspiel ist von einer Metathese geleistet worden. Das Bild wird durch die Wortspiele mit „mn“ „beständig“ und ,,mn.t“ „Bleiben“ zusätzlich ergänzt.

Das neunte Wortspiel ist zwischen „Imn“ „Amun“ und „mniw“ "30 „Hirte“ anzusetzen, das in einer ganzen Reihe von Beispielen präsentiert werden kann. Das erste Beispiel stammt aus der Verbindung, ,p3 mniw nfr n3 ntr.w rmt, $p 3$ nw (r) nhh d. d.t, ntk mniw nfr bnr irt.t $r n=k^{631}$ (Dritte Zwischenzeit), wofür die Wiedergabe „Oh guter Hirte der Götter und der Menschen, der bis in die Ewigkeit sieht. Du bist der gute Hirte, „Dattel“ und „Milch“ ist dein Name“ am

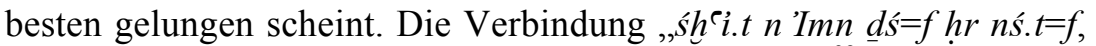

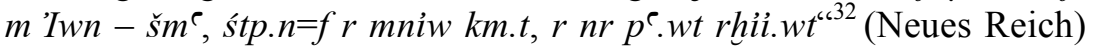
gehört als zweites Beispiel ebenfalls in diesen Rahmen, wofür die Wiedergabe ,die, die Amun selbst auf seinem Thron in Hermonthis erscheinen ließ, die er ausgewählt hat zum Hirten Ägyptens, zum Hirten der $p^{c} . w t$ - Leute und rhii.wt - Leute“" in Frage kommt. Die Worte haben auf die Königin Hatschepsut Bezug genommen. Die Palette kann durch das dritte Beispiel ,'Imn - Re, p3 mniw $n$ hr. $w-$ $n b . w n m h . w^{633}$ (Neues Reich) noch erweitert werden, das mit „Amun-

\footnotetext{
${ }^{29}$ Černý/Gardiner, Hieratic ostraca, Volume I, 39, 1.

${ }^{30}$ Zum Wort „mniw“ „Hirte“ vgl. Dieter Müller, Der gute Hirte, Ein Beitrag zur Geschichte ägyptischer Bildrede, Zeitschrift für Ägyptische Sprache und Altertumskunde 86 (1961), 126-144.

${ }^{31}$ Mourad Zaki Allam, Papyrus Berlin 3031, Totentexte der 21. Dynastie mit und ohne Parallelen, Bonn 1992, 164.

${ }^{32}$ Urk. IV, 361, 16-362, 1.

${ }^{33}$ Georges Posener, La Piété Personelle avant l'Âge Amarnien, Revue d'Égyptologie 27 (1975), 195-210, hier 204/205.
} 
Re, der Hirte für alle Bedrängten“ übersetzt werden kann. Das vierte Beispiel schließlich wird durch ,Imn mniw, śdw3 ịh.w, hd whd r śm.w, hd mniw ih.w (r) śm.w. 'Imn hd=k whd $r$ ' $k$.w, hr nti $r=f^{\prime}$ 'Imn mniw bw $g 3 w . n=f^{634}$ (Neues Reich) gebildet, wofür die Wiedergabe „Amun, du Hirte, der die Rinder früh ausführt, der die Leidenden zum Kraute treibt! Der Hirte treibt die Rinder zum Kraut - Amun, du treibst den Leidenden zum Brot. Denn Amun ist ein Hirte, der nicht ermattet." in Betracht gezogen werden kann. Der Abfall von „w“ bleibt als erste Voraussetzung für das Wortspiel festzuhalten, über den z.B. Westendorf gehandelt hat ${ }^{35}$. Die Ursache für das Wortspiel lässt sich außerdem in einer Metathese erkennen.

Das zehnte Wortspiel zeichnet sich zwischen „Imn“ „Amun“ und , $m n w^{\prime \prime}$ „Denkmal“ ab, das im Personennamen ,Imn - $m-$ $m n w=f^{636}$ (Neues Reich) „Amun ist in seinem Denkmal“ betrachtet werden kann. Die Basis für das Wortspiel ist durch den Lautwandel zwischen , $i “$ und „, $w^{\prime \prime}$ gelegt worden, der in der ägyptischen Sprache regelmäßig wiederkehrt ${ }^{37}$. Das Wortspiel ist durch eine Metathese besiegelt worden.

Das elfte Wortspiel hat sich zwischen „Imn“ „Amun“ und "mnh.t" „Linnen“ zugetragen, das an einer Stelle vorzukommen

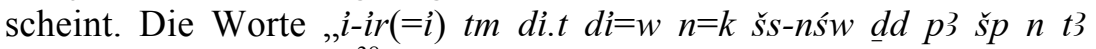
$m n h . t n$ 'Imn $m t w=k s^{6 / 38}$ (Spätzeit) sollen dazu in Erinnerung gerufen werden, die mit „Ich habe dir kein Byssos geben lassen, weil der Empfang des Linnens des Amun bei dir ist" übersetzbar sind. Die Erklärung für das Wortspiel bietet sich in Form des Lautwandels zwischen , $h^{\prime \prime}$ und , ," an, von welchem die Fachwelt z. B. durch Westendorf ${ }^{39}$ Kunde erlangt hat. Die zweite Stütze des Wortspiels hat eine Metathese gebildet. In seinem Kommentar zur Stelle schreibt

\footnotetext{
${ }^{34}$ Černý/Gardiner, Hieratic ostraca, Volume I, Pl. LXXXIX (oBritish Museum 5656a rto, 6-7).

${ }^{35}$ Westendorf, Grammatik der medizinischen Texte, 20.

${ }^{36}$ Yvan Koenig, Les ostraca hiératiques inédits de la Bibliothèque nationale et universitaire de Strasbourg, Documents de Fouilles de L'IFAO 33, Le Caire 1997, Pl. 75.

${ }^{37}$ Edel, Altägyptische Grammatik, 62 §142; Westendorf, Grammatik der medizinischen Texte, 21.

${ }^{38}$ Günther Vittmann, Der demotische Papyrus Rylands 9, Teil I, Text und Übersetzung, Ägypten und Altes Testament 38, Wiesbaden 1998, 162 (Text)/163 (Übersetzung).

${ }^{39}$ Westendorf, Grammatik der medizinischen Texte, 27-28/36.
} 
Vittmann, dass die Herstellung des ,mnh.t $t^{\text {“ }}$ - Leinens in den Tempelwerkstätten erfolgt sein könnte. Die enge Verbindung würde durch das Wortspiel noch deutlicher zum Ausdruck gebracht.

Das zwölfte Wortspiel lässt sich zwischen ,Imn“ „Amun“ und „mhn“" „Milchkanne“ zeigen, das einige Male gebildet worden ist. Der immer gleiche Satz lautet dabei ,p3 ihii $n$ p3 $m h n n$ ' $I m n^{\text {‘40 }}$ (Spätzeit), für den die Übersetzung „der Stall der Milchkanne des Amun“ passabel erscheint. Die Erklärung des Wortspiels könnte theoretisch über den Lautwandel zwischen , $i$ “ und , $h h^{\text {“ }}$ laufen, der bisher allerdings nur in ägyptisch - semitischer Richtung dokumentiert zu sein scheint ${ }^{41}$. Die Entstehung des Wortspiels hängt außerdem von einer Metathese ab. Der „Stall der Milchkanne des Amun“ wird von Spiegelberg $^{42}$ als Örtlichkeit im Tempelland des thebanischen Amun interpretiert.

Das dreizehnte Wortspiel kann zwischen „Imn“ „Amun“ und "mtrn“ „Weg“ dingfest gemacht werden, das gleich doppelt belegt ist. Das erste Beispiel lässt sich in „Imn $h r m \underline{r} n^{“ 43}$ (Neues Reich) erblicken, das mit „Amun auf der Straße“ wiederzugeben ist. Die

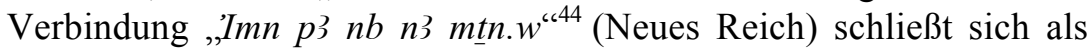
zweites Beispiel an, für das die Wiedergabe „Amun, der Herr der Wege“ gewählt werden kann. In der Erklärung des Wortspiels müssen zwei Faktoren berücksichtigt werden. Der Lautwandel zwischen , $i^{\text {“ }}$ und,$t^{\prime \prime}$ hat als erste wichtige Ursache zu diesem Wortspiel beigetragen, $\mathrm{zu}$ dem an anderem Ort eine eigene Untersuchung erscheinen wird ${ }^{45}$. Die zweite Vorraussetzung hat aus einer Metathese bestanden.

Das vierzehnte Wortspiel stellt sich zwischen „Imn“"Amun“ und „rmn“ „tragen“ ein, für das es zumindest ein Beispiel gegeben

\footnotetext{
${ }^{40}$ George Robert Hughes, Saite Demotic Land Leases, Studies in Ancient Oriental Civilizations 28, Chicago 1952, 45/51/68.

${ }^{41}$ Aaron Ember, Semito-Egyptian sound changes, Zeitschrift für Ägyptische Sprache und Altertumskunde 49 (1967/Neudruck der Ausgabe 1911), 87-94, hier 89.

${ }^{42}$ Wilhelm Spiegelberg, Zwei Kaufverträge aus der Zeit des Königs Harmachis (Papyrus Carnarvon I und II), in: Recueil de Travaux relatifs a la Philologie et a l'Archéologie Égyptiennes et Assyriennes 35 (1913), 150-161, hier 159.

${ }^{43}$ Sven P. Vleeming, Papyrus Reinhardt, An Egyptian land list from the tenth century B.C., Hieratische Papyri aus den Staatlichen Museen zu Berlin II, Berlin 1993, 18.

${ }^{44}$ Labib Habachi, Five Stelae from the Temple of Amenophis III at Es-Selu' now in the Aswan Museum, Kusch 8 (1960), 45-52, hier 47.

${ }^{45}$ Stefan Bojowald, Acta Orientalia (im Druck).
} 


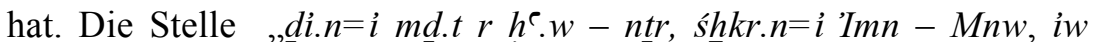
$r m n . n=i$ 'Imn $m h \underline{h}=f$, w $t$ ts. $n=i \dot{M} n r$ htiw $=f^{646}$ (Neues Reich) hat hier besondere Beachtung verdient, die mit „Ich habe Öl auf die Gottesglieder gegeben und den Amun - Min geschmückt. Ich habe Amun an seinem Fest getragen und Min auf seine Treppe gehoben.“ $\mathrm{zu}$ übersetzen ist. Die Verantwortung für die Entstehung des Wortspiels ist dem Lautwandel zwischen , ," und , "i" zuzuschreiben, der im Ägyptischen nicht gerade selten begegnet ${ }^{47}$.

Das fünfzehnte Wortspiel hat zwischen „Imn“ „Amun“ und ,śmn“ „festsetzen“ stattgefunden, das in einem Fall aufgetaucht ist. Das Interesse richtet sich hierbei auf das Satzgefüge , $d d m d w$ in pśd.t

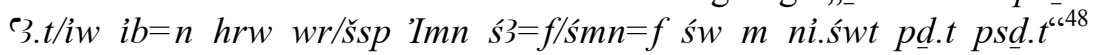
(Spätzeit), wofür die Übersetzung „Es spricht die große Neunheit: Unser Herz ist gar froh, denn Amun empfängt seinen Sohn und setzt ihn fest als König über die neun Bogenvölker.“ bemüht werden kann. Die Basis des Wortspiels ist in der Ähnlichkeit von , ,s“ und , , Hieratischen zu suchen.

Das sechzehnte Wortspiel ist zwischen „Imn“ „Amun“ und „Śmn“ „Nilgans“ vonstatten gegangen, für das sich mehrere Beispiele aufzählen lassen. Die erste Erwähnung soll das Beispiel „Imn p3 śmn $n d m$ hrrw"49 „Amun, die śmn - Gans mit süßer Stimme“ erhalten. Die Rede muss sodann auf das Beispiel ,śmn $n f r$ n 'Imn ${ }^{\text {“50 }}$ (Neues Reich) „schöne śmn - Gans des Amun“ gebracht werden. Die Wirkung der Wortspiele ist wieder durch die Ähnlichkeit von , „s“ und , „'“ im Hieratischen herbeigeführt worden. Die Verehrung der ,śmn“ - Gans als heiliges Tier des Amun darf hier stillschweigend als bekannt vorausgesetzt werden ${ }^{51}$. Der enge Bezug zwischen dem Gott und

\footnotetext{
${ }^{46}$ Urk. IV, 1031, 3-5.

${ }^{47}$ Westendorf, Grammatik der medizinischen Texte, 32.

${ }^{48}$ Hermann Junker/Erich Winter, Das Geburtshaus des Tempels der Isis in Philä, Wien 1965, 107.

${ }^{49}$ Waltraud Guglielmi/Johanna Dittmar, Anrufung der persönlichen Frömmigkeit auf Gans- und Widder-Darstellungen des Amun, in: Ingrid Gammer-Wallert (Ed.), Gegengabe, Festschrift für Emma Brunner-Traut, Tübingen 1992, 141.

${ }^{50}$ KRI I, 379, 13.

${ }^{51}$ Charles Kuentz, L 'oie du Nil (Chenalopex Aegyptiaca) dans l'antique Égypte, Lyon 1926, 48; Sethe, Amun und die acht Urgötter, 26; G. A. Wainwright, Some Aspects of Amun, in: The Journal of Egyptian Archeology 20 (1934), 139-153, hier 149; Eberhard Otto, Lexikon der Ägyptologie I, 239, s. v. Amun; Lothar Störk,
} 
seinem Begleitvogel wird durch die Wortspiele noch verstärkt. Das Wortspiel hat sich auch auf den Personennamen ,śmn.t-Imn “52 (Mittleres Reich) „Gans des Amun“ erstreckt. Die weibliche Form der Gänsebezeichnung lässt sich auch in anderem Kontext nachweisen ${ }^{53}$.

Das Ende des Artikels soll für ein abschließendes Fazit genutzt werden. Die Wortspiele mit „Imn“" „Amun“ haben eine unerwartet hohe Zahl ergeben. Die Häufigkeit der Wortspiele ist wohl im Zusammenhang mit der herausgehobenen Stellung des Götterkönigs $\mathrm{zu}$ sehen. In kleinem Maßstab ist dessen Person so auch mit sprachlichen Mitteln gehuldigt worden. Im Prinzip lässt sich der gleiche Fall bei anderen Göttern beobachten. Die Zahl der dortigen Wortspiele scheint zwar niedriger als bei Amun zu liegen. Die vorsichtige Schätzung des Verf. hat zu je fünf bis sechs Beispielen bei Re, Sachmet und Anubis geführt. Die Situation wird aber ähnlich zu interpretieren sein. Die Andeutungen in diesem Artikel mögen vorläufig genügen. Der Autor hofft bei späterer Gelegenheit auf das Thema zurückzukommen.

Lexikon der Ägyptologie IV, 484, s. v. Nilgans; Sven P. Vleeming, The gooseherds of Hou, Studia Demotica III, Leuven 1991, 2.

${ }^{52}$ Hermann Ranke, Die ägyptischen Personennamen, Band II, Glückstadt 1952, 185.

${ }^{53}$ Selim Hassan, Excavations at Giza, with Spezial Chapters on Methods of Excavation, the False - door, and other Archaeological and Religious Subjects, Vol. V 1933-1934, Excavations of The Faculty of Arts, Fouad I. University, Service des Antiquités de 1' Égypte, Cairo 1944, 117; Karl Jansen-Winkeln, Ägyptische Biographien der 22. und 23. Dynastie, Ägypten und Altes Testament 8, Wiesbaden 1985, 16312. 
